\title{
Modeling the Flow Rate of Pneumatic Driving Micro-droplet Jetting Process

\author{
Cong-ping Chen ${ }^{1, a^{*}}$, Jie-guang Huang ${ }^{1, b}$
}

${ }^{1}$ College of Mechanical \&Power Engineering of China Three Gorges University, Yichang 443002, HuBei, China

aemail: mechencp@163.com

bemail: jieguangh@qq.com

Keywords: Droplet jetting, Power law non Newtonian fluid, Flow rate model.

Abstract. Droplet jetting technology is widely used in 3D printing. In which process, the flow rate of material extrusion from the nozzle is one of the keys that affect the printing performance, such as the droplet size and its uniformity. In this paper, a model was developed to predict the jetting flow rate. First, by analyzing the dynamics of the power law fluid flowing in circular tube, a flow rate model of tube flow is obtained. Second, the dynamics of fluid flowing through a cone tube is obtained. Finally, a set of jetting flow rate model is developed by combining the results delivered in previous two steps. The model's validity was verified by some numerical simulations, the results showed that the model developed in this paper can predict the jetting flow rate effectively.

\section{Introduction}

Droplet jetting is a kind of technologies that use some drive styles such as piezoelectric ceramic, pulse gas, screw et al. to extrude the fluid materials out from the jet precisely. It has been widely used in many fields such as 3D printing [1], tissue scaffold preparation [2,3], cell printing [4], integrated circuit packaging [5] .Droplet jetting technologies can be classified into pneumatic type, piezoelectric ceramic type, cylinder rod type and screw pump based on driving source. However, pneumatic droplet jetting technology takes major possession of market share because of its convenient maintenance and low cost performance.

The structure schematic diagram of a typical pneumatic droplet jet is shown as Fig.1, which utilizes gas pulse to drive fluid material in the jet to eject in the form of a droplet. The flow rate of the nozzle is the key factor that affects the volume, size and uniformity of the droplet directly. At present, the researches of jetting processes almost focus on mechanics analysis using classic theoretical, and combining numerical modeling or experiment to verify the accuracy. An operable physical analysis model completed with a certain universality hasn't been obtained. Hence it`s limited in practical application. Xiao Yuan et al. [6] investigated the influence of technological parameters on the uniformity of the droplet using true experimental method in the processes of pressure driving molten metal to jet droplets. They obtained the statistical data in the condition of giving technological parameters. But the universality needs to be validated. Jun Luo, Le-hua Qi et al. [7] proposed a 2D axisymmetric model to simulate the droplet generation. They applied a proprietary pneumatic DOD (drop on demand) generator to conduct the droplet generation experiments. The simulation observations agreed well with the experimental observations in droplet pattern, breakup length and droplet diameter in single droplet generation process. S. Cheng and S. Chandra [8] designed a pneumatic droplet generator to produce water droplets on demand. They photographed droplets as they emerged from the nozzle, and recorded pressure fluctuation in the chamber. Besides they determined the duration of the pressure pulse required to generate a single drop. Hung-Ju Chang and Ming Hsiu Tsai et al. [9] found that the pressure-time relationship at the nozzle is the dominant factor that determines the droplet formation behavior. A numerical model was employed to study the correlations of droplet formation behavior between pressure-time relationship and the following three types: single droplet, column droplet and molten lead-free solder can not be jetted. 
The pneumatic droplet jetting process was studied in this paper. It has modeled the flow rate of droplet injection, which can be applied to a variety of power-law fluid. Two dynamic models of fluid flow in liquid chamber ( $L_{2}$ as shown in Fig.1) and nozzle ( $L_{3}$ as shown in Fig.1) are established. Then a flow rate model of fluid jetting is obtained by combining the two models. The model is validated by some numerical simulations.

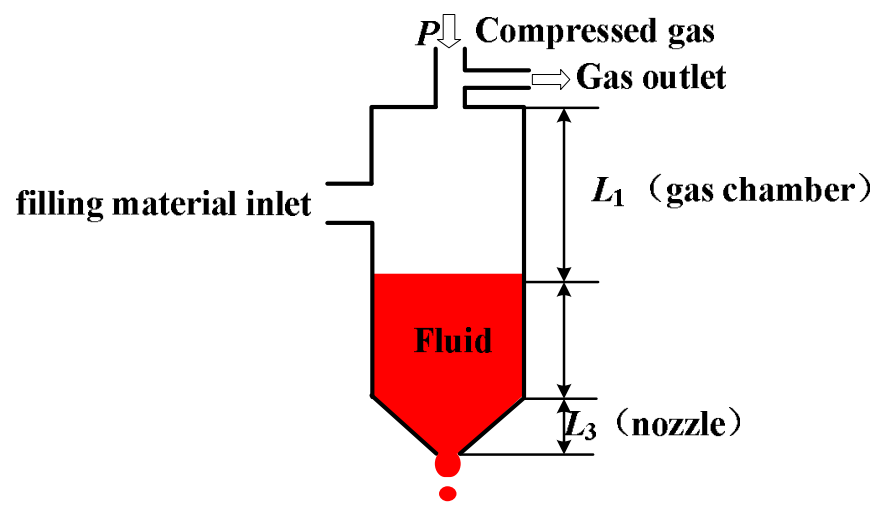

Fig.1. Structure schematic of pneumatic droplet jet

\section{Dynamic Modeling}

As the geometrical characteristic of nozzle and liquid chamber are different from each other, then the model is divided into two parts, the liquid chamber (constant diameter tube) flow rate model and the nozzle (cone tube) flow rate model.

Liquid Chamber Flow Rate Model. As shown as Fig.2, the pressure $P_{r}$ impacted on fluid in the cylinder liquid chamber, the reaction force $P_{s}$ on the interface of liquid chamber and nozzle, and the frictional force between fluid and tube wall are in a state of stress equilibrium. It can be represented by the momentum conservation equation and mass continuity equation

$$
\begin{aligned}
& \rho \frac{D \mathbf{U}}{D t}-\nabla P-\nabla \boldsymbol{\tau}-\rho g=0, \\
& \frac{\partial \rho}{\partial t}+(\nabla \rho \mathbf{U})=0 .
\end{aligned}
$$

In the cylindrical coordinate, $\nabla=\partial / \partial r+\partial / \partial z, \mathbf{U}=\left[\begin{array}{lll}u_{r} & u_{\theta} & u_{z}\end{array}\right]^{T}$ is velocity vector, $\rho$ is the fluid density, $\boldsymbol{\tau}$ is the shearing stress.

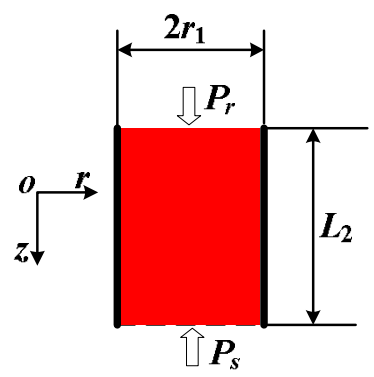

Fig.2 Stress schematic of fluid in the liquid chamber

For the fluid in the liquid chamber, the following assumptions have been given: (1) The process is heat insulation. (2)The fluid is incompressible (that is $\partial \rho_{L} / \partial t=0$ ). (3) The fluid is steady laminar flow (that is $\partial u_{z} / \partial z=0$ ). (4) The gravity is neglected (that is $\rho g=0$ ). Besides, as the liquid chamber is an axisymmetric circular tube, that means $u_{\theta}=0$. Base on the assumptions above, the continuity equation can be simplified as following

$$
\frac{\partial}{\partial r}\left(r u_{r}\right)=0 \text {. }
$$

There is $r u_{r}=C$ ( $C$ is constant). The wall boundary condition is $\left.u_{r}\right|_{r=r_{1}}=0$, thus $C=0, u_{r} \equiv 0$. 
Then equation (1) can be simplified as

$$
\frac{\partial P}{\partial z}+\frac{1}{r} \frac{\partial}{\partial r} r\left(\tau_{r z}\right)=0
$$

For the power law fluid, the shear stress is

$$
\tau_{r z}=k \gamma \&=k\left(-\frac{d u_{z}}{d r}\right)^{n} \text {. }
$$

Where $k, \gamma$ are viscosity and shearing rate, respectively. Substitute Eq. (5) to Eq. (4), and integrate $r$. According to the boundary conditions $u_{z}=\left.0\right|_{r=r_{1}}, u_{z}<\left.\infty\right|_{r=0}$ one can get

$$
u_{z}=\frac{n}{n+1}\left(\frac{P_{r}-P_{s}}{2 L_{2} k}\right)^{\frac{1}{n}}\left(r_{1}^{\frac{n+1}{n}}-r^{\frac{n+1}{n}}\right) \text {. }
$$

Then the flow rate $Q_{L_{2}}$ of liquid chamber can be presented as

$$
Q_{L_{2}}=\int_{0}^{r_{1}} 2 \pi r u_{z} d r=\frac{n \pi r_{1}^{3}}{3 n+1}\left(\frac{r_{1}}{2 L_{2} k}\right)^{\frac{1}{n}}\left(P_{r}-P_{s}\right)^{\frac{1}{n}}
$$

However, $P_{s}$ in equation (7) can't be measured. So that, the flow rate of orifice can't be evaluated directly according to flow rate conversation principle. Hence, it can be eliminated by combining the dynamics model of fluid flowing through the nozzle.

The geometry parameters of nozzle are shown as Fig.3. Intercepting arbitrary a cone fluid element along the nozzle axis is shown as Fig.4.

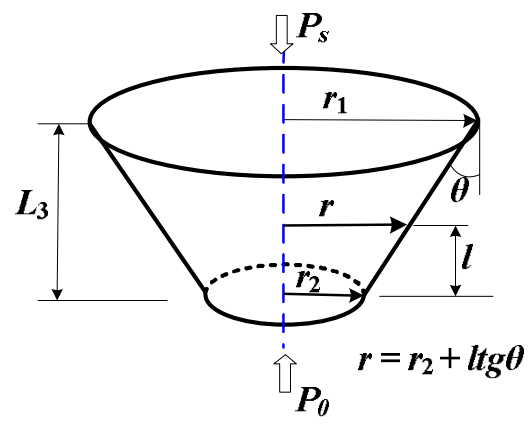

Fig.3 Geometry parameters of nozzle

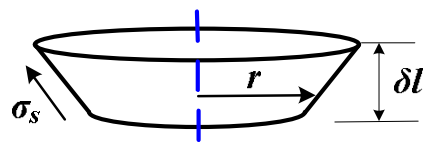

Fig.4. Schematic of stress of fluid element

The thickness is $\delta l \rightarrow 0$, and the radius is $r$. The micro element is stress equilibrium along its axis. That is

$$
\left(\delta P_{s}\right) \pi r^{2}=2 \pi r(\delta l) \sec \theta \tau_{r z} \cos \theta .
$$

There is

$$
\delta P_{s}=\frac{2 \tau_{r z}(\delta l)}{r} \text {. }
$$

As $\delta l=\delta r / \operatorname{tg} \theta, \tau_{r z}=k \gamma \&$, and the connection between shearing rate and flow rate $Q_{L_{3}}$ is

$$
\alpha=\frac{3 n+1}{4 n} \cdot \frac{4 Q_{L_{3}}}{\pi r^{3}} .
$$

Substitute it to Eq. (9). There is

$$
\delta P_{s}=\frac{2 k}{\operatorname{tg} \theta}\left(\frac{3 n+1}{4 n} \cdot \frac{4 Q_{L_{3}}}{\pi}\right)^{n} \frac{\delta r}{r^{(1+3 n)}} .
$$

Integrate Eq. (11). There is

$$
P_{s}=\frac{2 k}{\operatorname{tg} \theta}\left(\frac{3 n+1}{4 n} \cdot \frac{4 Q_{L_{3}}}{\pi}\right)^{n} \int_{r_{2}}^{r_{1}} \frac{1}{r^{(1+3 n)}} d r=\frac{2 k}{3 n r_{2}^{3 n} \operatorname{tg} \theta}\left(\frac{3 n+1}{4 n} \cdot \frac{4 Q_{L_{3}}}{\pi}\right)^{n}\left[1-\left(\frac{r_{2}}{r_{1}}\right)^{3 n}\right] .
$$


As a result, the volume flow rate of the jet is

$$
Q_{L_{3}}=\frac{n \pi r_{2}^{3}}{3 n+1}\left[\frac{3 n t g \theta P_{s}}{2 k\left[1-\left(r_{2} / r_{1}\right)^{3 n}\right]}\right]^{1 / n} \text {. }
$$

But $P_{s}$ in equation (13) is also unknown and immeasurable.

Flow Rate Model. As the fluid is assumed to be incompressible, there must be $Q_{L_{2}}=Q_{L_{3}}$. So that, combining (7) and (13), and eliminating $P_{s}$, there is

$$
Q_{L_{3}}=\frac{n \pi r_{1}^{3}}{3 n+1}\left(\frac{3 n r_{1} t g \theta P_{\mathrm{r}}}{6 k n L_{2} \operatorname{tg} \theta+2 k r_{1}\left[\left(r_{1} / r_{2}\right)^{3 n}-1\right]}\right)^{1 / n} \text {. }
$$

Define $K=\frac{n \pi r_{1}^{3}}{3 n+1}\left(\frac{3 n r_{1} \operatorname{tg} \theta}{6 k n L_{2} \operatorname{tg} \theta+2 k r_{1}\left[\left(r_{1} / r_{2}\right)^{3 n}-1\right]}\right)^{1 / n}$. The droplet jetting flow rate $Q$ is

$$
Q=K P_{\mathrm{r}}^{\frac{1}{n}}
$$

In the duration $t_{d}$ of pressure impact, the ejecting volume $V_{L}$ is

$$
V_{L}=\int_{0}^{t_{d}} Q d t
$$

\section{Numerical Simulation}

Simulation Model. The jetting flow rate and driven pressure are dynamic parameters and can not be measured online. Hence this paper is plane to validate the model with numerical modeling method. A CFD simulation Microsoft FLUENT 6.3 is used to simulate the droplet jetting processes. And the numerical modeling observations are taken as reference to evaluate the prediction accuracy of the model. The jet parameters in numerical modeling are as follows: $L_{1}=70 \mathrm{~mm}, L_{2}=10 \mathrm{~mm}$, $L_{3}=43 \mathrm{~mm}, r_{1}=15 \mathrm{~mm}, r_{2}=0.16 \mathrm{~mm}$. A rectangular pressure pulse impacted on the entrance of the gas tube is simulated as gas source. The pulse gas is set to ideal gas. The boundary condition of the gas inlet is set to pressure inlet. While it is gassing, the boundary condition is set pressure outlet. The fluid is set to incompressible non- Newtonian fluid, and the flow property is laminar flow. The density is $1780 \mathrm{~kg} / \mathrm{m}^{3}$. The jetting process is thermostatic (it is set to $300 \mathrm{~K}$ ). The gravity acceleration is $g=9.81 \mathrm{~N} / \mathrm{s}^{2}$. The pressure and velocity coupling field is calculated using PISO algorithm of separated solver.

Simulation Observations. Four different kinds of fluid are used to validate the flow rate model of orifice. The correlation jetting parameters are shown in table 1. According to different kinds of fluid, the amplitude $(P)$ and pulse width $\left(t_{d}\right)$ of the pressure pulse impacted are different too. In table $1, t_{s}$ is the time necking, and $t_{b}$ is the time droplet breaking away from jet. They are obtained by numerical modeling. The gas-fluid constitutional diagrams, at different time, of fluid in table 1 are shown as Fig.5.

Table 1 The droplet jetting parameters of different fluid

\begin{tabular}{cccccccc}
\hline & $P(P a)$ & $T(m s)$ & $t_{d}(m s)$ & $k\left(P a \cdot s^{n}\right)$ & $n$ & $t_{s}(m s)$ & $t_{b}(m s)$ \\
\hline A & 4500 & 60 & 2 & 7.5 & 0.3 & 14 & 26 \\
B & 4000 & 60 & 2.5 & 8 & 0.3 & 15 & 28 \\
C & 25000 & 80 & 6 & 0.005 & 1.5 & 13 & 53 \\
D & 24000 & 100 & 10 & 0.01 & 1.5 & 21 & 63 \\
\hline
\end{tabular}

The research indicates that the fluid can't be extruded, or be extruded in the type of jet, or exist satellite droplets while the amplitude $(P)$ and pulse width $\left(t_{d}\right)$ aren't configured properly in the condition of the fluid properties and jet geometry parameters set. It`s hard to configure the values of amplitude $(P)$ and pulse width $\left(t_{d}\right)$ to obtain perfect droplets flow that is continuous, smooth and no 
satellite droplet. This paper had been tried lots of times before obtained comparative ideal parameters configuration. It`s very hard to carry out quantitative jetting referred to model. This problem is one of generally acknowledged difficulties. And it will be continued in the follow-up studies.

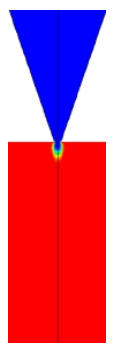

$5 m s$

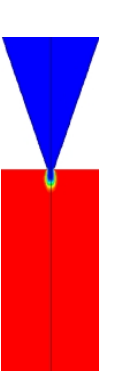

$5 m s$

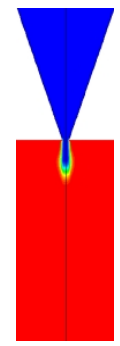

$10 m s$

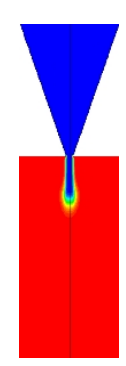

$13 m s$

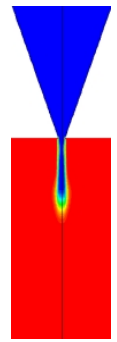

$19 m s$

(a)

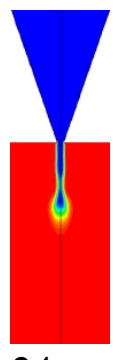

(c)

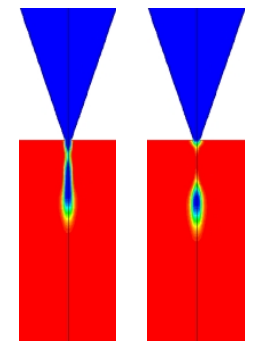

$22 m s \quad 26 m s$

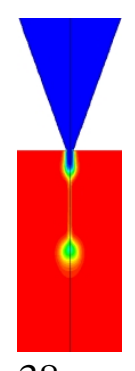

$38 m s$

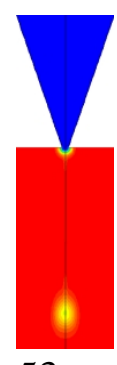

$53 m s$

Fig.5 Constitutional diagram of droplet jetting process. (a) Fluid A; (b) Fluid B;

(c) Fluid C; (d) Fluid D.

According to table 1 and Fig.5, we found that the effect of index $n$ on the formation of droplet is relative great. In the condition of $0<n<1$ (fluid A, B), the respective major viscosity fluid only need minor pressure and actuation duration to be formed droplet. In the condition of $n>1$ (fluid C, D), even if the fluid viscosity is minor, the pressure growth in series, the droplet formation period and breaking up time are all incrementing. It costs more time from necking to breaking up. Droplets are easy to stretch longer, and are very easier to form a column.

In order to verify the availability of the flow rate model, substitute $P_{r}$ to equation (15) to calculate the volume flow rate of nozzle in a pressure period. The value of $P_{r}$ can be obtained using monitoring function of FLUENT software in numerical modeling. The consequences of comparing calculation results with simulation observations are shown as Fig.6. Where $Q_{m}$ is the calculation result of flow rate using theoretical model, while $Q_{n}$ is the simulation result of flow rate. We only pick the simulation data before $t_{s}$ moment to verify the flow rate model. Because with the decrease of the pressure in the gas chamber, the shear rate of fluid is decreased too after $t_{s}$ moment. The main impact on the fluid is the fluid elastic force and the surface tension. The power law equation $\tau_{r z}=k \gamma \mathcal{Q}$ is not accuracy any more. As a result, the flow rate model is not accuracy any more too.

According to Fig.6, we found that the results calculated by the flow rate model agree well with the simulation observations. The $Q-t$ curves of fluid A and B are different from the $Q-t$ curves of fluid $C$ and $D$. In the condition of $0<n<1$, the growth rate is almost identical with the decay rate of the curves as Fig.6 (a), (b) shown. While, in the condition of $n>1$, the curve increased rapidly first, then decreased gradually as Fig.6 (c), (d) shown. 


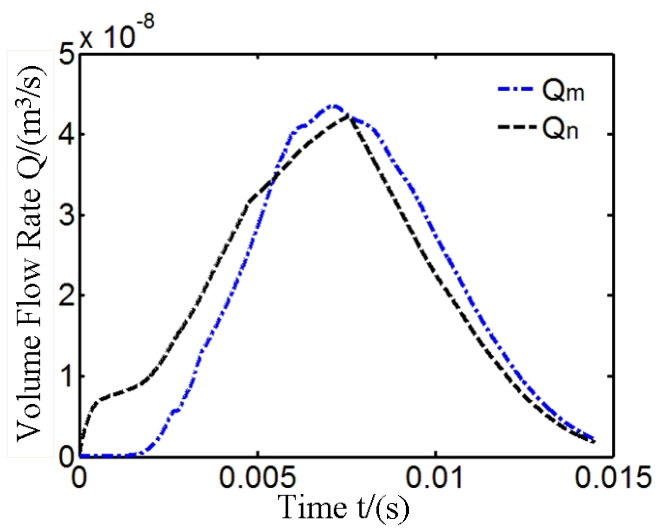

(a)

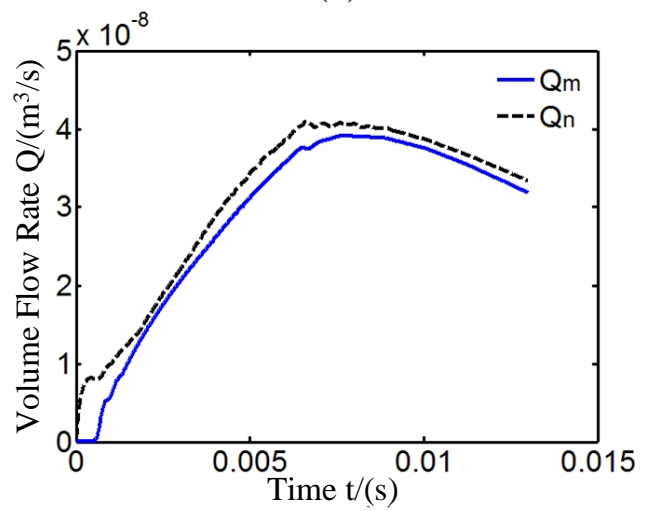

(c)

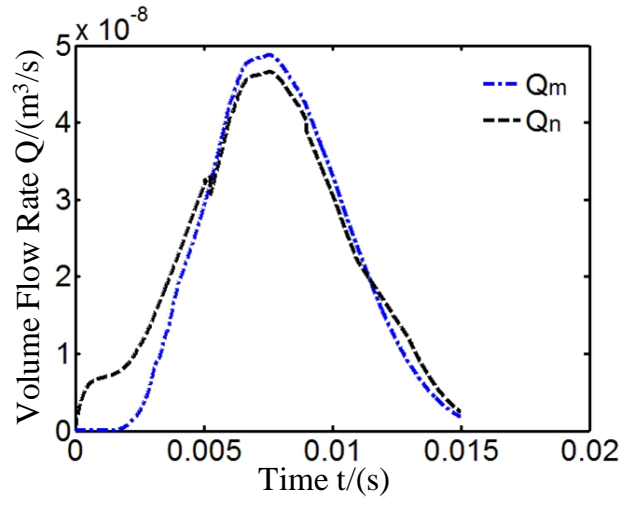

(b)

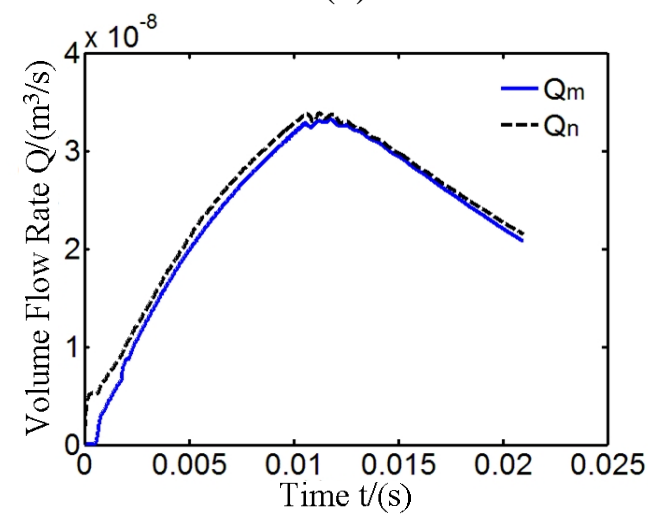

(d)

Fig.6 $Q-t$ curve in a jetting period. (a) Fluid A; (b) Fluid B; (c) Fluid C; (d) Fluid D.

Base on the simulation observations, the error of flow rate model are defined as

$\bar{\varepsilon}_{Q}=\left|\frac{Q_{m}-Q_{m}}{Q_{m}}\right| \times 100 \%$.

Where $\bar{\varepsilon}_{Q}$ is the error of flow rate model. The error $\bar{\varepsilon}_{Q}$ of fluid A, B, C, D are shown as table 2 .

Table 2 The value of $\bar{\varepsilon}_{Q}$ of different kinds of fluid (\%)

\begin{tabular}{ccccc}
\hline & $\mathrm{A}$ & $\mathrm{B}$ & $\mathrm{C}$ & $\mathrm{D}$ \\
\hline $\bar{\varepsilon}_{Q}$ & 4.79 & 6.93 & 7.12 & 4.32 \\
\hline
\end{tabular}

According to Table 2 , the error of flow rate model is under $8 \%$. We hold that the flow rate model can exactly predict the flow rate of a droplet jetting system described above.

\section{Summary}

$\dagger \quad$ This paper modeled the flow rate of power law non-Newtonian fluid extruded out of the orifice in droplet jetting process. The model can be applied to a variety of power law non-Newtonian fluid.

$\dagger$ We have validated the accuracy of the model effectively with numerical simulation observations. The $Q-t$ curves of four kinds of fluid calculated by model and simulated by software were drew to analysis the error of the model we built. We found that the results calculated by the flow rate model agree well with the simulation observations.

$\dagger$ The error of flow rate model is under $8 \%$. We hold that the flow rate model can exactly predict the flow rate of a droplet jetting system described above. 


\section{Acknowledgment}

The research was supported by the National Natural Foundation of China (No. 51475266) and Postgraduate Research Innovation Fund of China Three Gorges University (No. 2015CX039).

\section{References}

[1] Madhusudan Singh, Hanna M. Haverinen, Parul Dhagat, Ghassan E. Jabbour, Inkjet printing-process and its applications, Advanced Materials, 22(2010) 673-685.

[2] Andres Diaz Lantada, Pilar Lafont Morgado, Rapid Prototyping for Biomedical Engineering Current Capabilities and Challenges, Annu. Rev. Biomed. Eng. 14(2012)73-96.

[3] Thomas Billiet, Elien Gevaert, Thomas De Schryver, Maria Cornelissen, Peter Dubruel, The 3D printing of gelatin methacrylamide cell-laden tissue-engineered constructs with high cell viability, Biomaterials, 35(2014)49-62.

[4] Falguni Pati, Dong-Heon Ha, Jinah Jang, Hyun Ho Han, Jong-Won Rhie, Dong-Woo Cho, Biomimetic 3D tissue printing for soft tissue regeneration, Biomaterials, 62( 2015)164-175.

[5] CHEN Congping, LI Hanxiong, DING Han, Model-based Control of Time pressure Dispensing Process, MACHINE TOOL\&HYDRAULICS, 35(2007) 173-175.

[6] XiaoYuan, Huang Yachao, Jiang Long, Chen Lan, Research on Micro-droplet Controllable Jetting in Fine Circuit Formation Using Jet Printing and Chemical Deposition. China Mechanical Engineering, 26(2015)1806-1809.

[7] Jun Luo, Le-hua Qi, Ji-ming Zhou, Xiang-hui Hou, He-jun Li, Modeling and characterization of metal droplets generation by using a pneumatic drop-on-demand generator, Journal of Materials Processing Technology, 212(2012)718-726.

[8] S. Cheng, S. Chandra, A pneumatic droplet-on-demand generator. Experiments in Fluids, 34(2003)755-762.

[9] Hung-Ju Chang, Ming Hsiu Tsai, Weng-Sing Hwang, The simulation of micro droplet behavior of molten lead-free solder in inkjet printing process and its experimental validation, Applied Mathematical Modeling, 36(2012)3067-3079. 